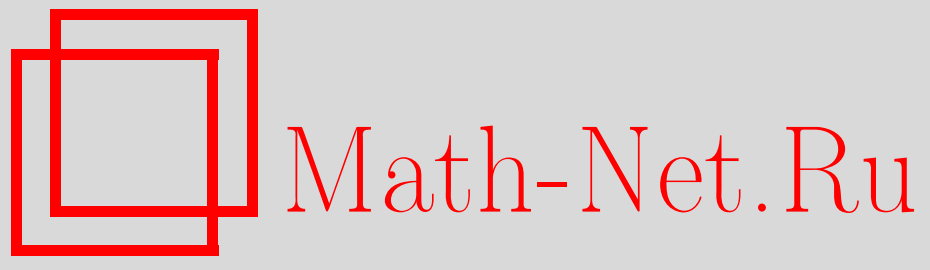

С. Ю. Новак, О точности оценивания характеристик распределений с тяжелыми хвостами, Теория вероятн. и ее примен., 2013, том 58, выпуск 3, 598-607

DOI: https://doi.org/10.4213/tvp4530

Использование Общероссийского математического портала Math-Net.Ru подразумевает, что вы прочитали и согласны с пользовательским соглашением http://www . mathnet.ru/rus/agreement

Параметры загрузки:

IP : 54.81 .137 .203

26 апреля 2023 г., 14:19:11

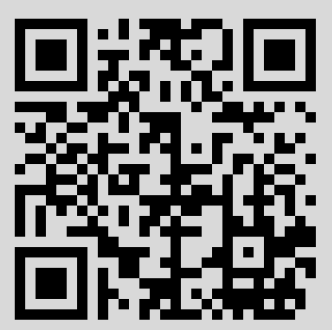


(c) 2013 г.

HOBAK C. Ю.*

\section{О ТОЧНОСТИ ОЩЕНИВАНИЯ ХАРАКТЕРИСТИК РАСПРЕДЕЛЕНИЙ С ТЯЖЕЛЫМИ ХВОСТАМИ}

В работе предложен простой метод получения непараметрических нижних границ точности статистического оценивания характеристик распределений с тяжелыми хвостами. Установлены нижние границы среднеквадратических ошибок оценок показателя скорости убывания хвоста распределения, константы хвоста распределения и экстремального квантиля. Из полученных результатов следует, что нормирующие последовательности робастных оценок должны специфическим образом зависеть от показателя скорости убывания хвоста распределения и константы хвоста распределения.

Ключевые слова и фразы: нижние границы, распределения с тяжелыми хвостами.

1. Введение. Распределения с тяжелыми хвостами возникают естественным образом в финансах, метеорологии, гидрологии, теории передачи информации и т.д. [10], [20]. В частности, замечено, что цены, замеренные через короткие промежутки времени, часто представляются случайными величинами с тяжело-хвостовыми распределениями [5], [10], [15].

Случайная величина $X$ имеет распределение с тяжельм правым хвостом (РТХ), если

$$
\mathbf{P}(X \geqslant x)=L(x) x^{-\alpha} \quad(x>0),
$$

где $\alpha>0$, а (неизвестная) функция $L$ медленно меняется на $+\infty$ :

$$
\lim _{x \rightarrow \infty} \frac{L(x t)}{L(x)}=1 \quad(\forall t>0) .
$$

Класс распределений с тяжелым правым хвостом мы обозначим через $\mathscr{H}$.

Число $\alpha$ в (1) называется показателем скорости убьвания хвоста распределения (ПСУХ). Это основная характеристика хвоста распределения. Если $L(x)=$ $C+o(1)$, то $C$ называется константой хвоста распределения.

Задача оценивания ПСУХ оказалась непростой; она привлекала внимание исследователей на протяжении десятилетий (см. [10], [18], [20] и ссылки там). Установлена состоятельность и асимптотическая нормальность ряда оценок ПСУХ (см. [10], [18]). Тем не менее вопрос о нижней границе средне-квадратической ошибки оценок ПСУХ в общем случае оставался открытым.

Нижнюю границу средне-квадратической ошибки в случае регулярного параметрического семейства распределений устанавливает знаменитое неравенство ФрешеРао-Крамера; нижние границы средне-квадратической ошибки известны также для параметрических семейств с определенными нерегулярностями [14], [21].

Отметим, что $\mathscr{H}$ является непараметрическим классом распределений. В задачах непараметрического оценивания типичной представляется ситуация, когда оценка является функцией управляющего (мешающего) параметра (ср. с (15)). Это значительно усложняет задачу статистического оценивания.

* Middlesex University, MUBS, The Burroughs, London NW44BT, UK; e-mail: s.novak@mdx.ac.uk

1) Работа выполнена при поддержке гранта Лондонского математического общества. 
Первой работой, посвященной вопросу о нижних границах точности оценивания ПСУХ, является статья П. Холла и А. Вэлша [8], где был доказан следующий результат. Пусть $\mathscr{D}_{A}\left(\alpha_{0}, C_{0}, \varepsilon, b\right)$ - класс распределений на $(0 ; \infty)$ с плотностями

$$
f(x)=C \alpha x^{-\alpha-1}(1+r(x)),
$$

где $\sup _{x>0}|r(x)| x^{b \alpha} \leqslant A,\left|\alpha-\alpha_{0}\right| \leqslant \varepsilon,\left|C-C_{0}\right| \leqslant \varepsilon$. Обозначим через $\widehat{\alpha}_{n} \equiv$ $\widehat{\alpha}_{n}\left(X_{1}, \ldots, X_{n}\right)$ произвольную оценку ПСУХ, где $X, X_{1}, \ldots, X_{n}-$ независимые одинаково распределенные случайные величины, и пусть $\left\{z_{n}\right\}$ - произвольная последовательность положительных чисел. Если

$$
\lim _{n \rightarrow \infty} \sup _{F \in \mathscr{D}_{A}} \mathbf{P}_{F}\left(\left|\widehat{\alpha}_{n}-\alpha\right|>z_{n}\right)=0 \quad(\forall A>0)
$$

для некоторых $\alpha_{0}>0, C_{0}>0, b>0, \varepsilon>0$, то

$$
z_{n} \gg n^{-b /(2 b+1)}
$$

(более точно, Холл и Вэлш получили указанный результат для случайных величин $Y_{i}=1 / X_{i}$, где распределение случайных величин $X_{i}$ удовлетворяет (2)). Я. Бейрлант, К. Букьо и Б. Веркер [1] установили аналогичный результат для более широкого класса $\mathscr{P}$ распределений, но при условии, что оценки являются $O_{\mathbf{P}}(1)$ равномерно B $\mathscr{P}$.

Близкий результат принадлежит Й. Пфанцалю [19]. Пусть $\mathscr{D}_{b}-$ класс распределений с плотностями (2) такими, что $\sup _{x>0}|r(x)| x^{\alpha b}<\infty, \alpha>0$. Обозначим

$$
s_{n}\left(c, P_{0}\right)=\sup _{P \in \mathscr{P}_{n, c}}\left|\alpha_{P}-\alpha_{P_{0}}\right|,
$$

где $\alpha_{P}$ - показатель скорости убывания хвоста распределения $P, \mathscr{P}_{n, c}=\{P \in$ $\left.\mathscr{D}_{b}: d_{\mathrm{TV}}\left(P_{0}^{n} ; P^{n}\right) \leqslant c\right\}$ - окрестность распределения $P_{0} \in \mathscr{D}_{b}$ и $d_{\mathrm{TV}}-$ расстояние по вариации. Пфанцаль показал, что никакая оценка не сходится к $\alpha_{P}$ равномерно по $\mathscr{P}_{n, c}$ со скоростью лучшей, чем $s_{n}\left(c, P_{0}\right)$, при этом

$$
\inf _{0<c<1} c^{-2 b /(1+2 b)} \liminf _{n \rightarrow \infty} n^{b /(1+2 b)} s_{n}\left(c, P_{0}\right)>0 .
$$

Д. Донохо и Р. Лиу [3] предложили нижнюю границу точности оценивания ПСУХ в терминах модулей непрерывности $\Delta_{A}(n, \varepsilon)$, однако они не вычисляют $\Delta_{A}(n, \varepsilon) ;$ утверждение, что некое распределение с тяжелым хвостом стохастически доминирует все РТХ с тем же ПСУХ, предложено без доказательства. Х. Дреес [4] вывел асимптотику минимаксного риска для аффинных оценок ПСУХ и отметил возможность численного вычисления асимптотики минимаксного риска для неаффинных оценок. П. Холл и А. Вэлш [8] показали также, что $z_{n} \gg(\ln n) n^{-b /(2 b+1)}$, если в (3) $\widehat{\alpha}_{n}-\alpha$ заменить на $\widehat{C}_{n}-C$, где $C$ - константа хвоста распределения, а $\widehat{C}_{n}-$ ее произвольная оценка.

Среди непараметрических семейств тяжело-хвостовых распределений, рассматриваемых в литературе, отметим класс

$$
\mathscr{H}_{a, b, c, d}=\left\{\mathbf{P}: \mathbf{P}(X \geqslant x)=c x^{-1 / a}\left(1+d x^{-b / a}(1+o(1))\right)\right\}
$$

распределений на $(1 ; \infty)$, где $a=1 / \alpha>0, b>0, c \equiv c(a)>0, d \equiv d(a, b) \neq 0$ [17], [18]. Ряд других классов РTX, а также сравнение оценки Хилла и RE-оценки ПСУХ можно найти в [18]. Параметрическое семейство Парето

$$
\mathbf{P}(X \geqslant x)=x^{-1 / a} \quad(x \geqslant 1, a>0)
$$

можно считать «предельной точкой» класса $\mathscr{H}_{a, b, 1, d}$, когда второй индекс, $b$, стремится к бесконечности. Отметим, что

$$
\mathbf{E}\left(\frac{a_{n}^{*}}{a}-1\right)^{2}=\frac{1}{n}, \quad\left(\frac{a_{n}^{*}}{a}-1\right) \sqrt{n} \Rightarrow \mathscr{N}(0 ; 1) \quad(n \rightarrow \infty)
$$


в случае независимых одинаково распределенных наблюдений над распределением Парето (5), где $a_{n}^{*}=n^{-1} \sum_{i=1}^{n} \ln X_{i}$.

В настоящей работе предлагается простой способ получения непараметрических нижних границ средне-квадратической ошибки. В основе нашего подхода лежит лемма 1, представленная в п. 3. В следующем пункте мы применяем указанный подход к задачам непараметрического оценивания характеристик распределений с тяжелыми хвостами и выводим непараметрические нижние границы среднеквадратической ошибки оценок показателя скорости убывания хвоста распределения, константы хвоста распределения и экстремального квантиля. Нижние границы среднеквадратической ошибки оценок ПСУХ и экстремальных квантилей получены, насколько нам известно, впервые. Нижние границы увеличиваются при уменьшении $\alpha$, указывая, что задача оценивания становится сложнее для распределений с более тяжелыми хвостами. Из полученных результатов следует также, что нормализующие последовательности зависят специальным образом от ПСУХ и константы хвоста распределения. Доказательства отнесены в п. 3.

2. Нижние границы. Традиционный подход к получению непараметрических нижних границ предлагает выбрать «как можно больше функций распределений, отделенных друг от друга не менее, чем (небольшим расстоянием) $\delta>0 \gg[12],[13]$, [9]. Другой метод основан на построении двух «близких» функций распределения $F_{0}$, $F_{1}[6],[8],[22]$. Мы покажем ниже, что последний подход позволяет получать минимаксные нижние границы средне-квадратической ошибки оценок ПСУХ, константы хвоста распределения и экстремального квантиля.

Подмечено, что точность оценивания в случае непараметрического класса распределений обычно хуже, чем в случае регулярного параметрического семейства. Точность оценивания зависит от «размерности» класса возможных распределений: чем богаче класс, тем труднее задача выбора между близкими альтернативами, и, следовательно, тем хуже точность оценивания. Например, в задаче непараметрического оценивания плотности исследователь имеет дело с классом распределений, удовлетворяющим определенному условию гладкости; чем более гладкими являются плотности, тем выше точность оценивания [12], в то время как при отсутствии каких-либо предположений о гладкости распределений скорость убывания средне-квадратической ошибки может быть сколь угодно плохой [2].

Аналогично, класс $\mathscr{H}$ распределений с тяжелым правым хвостом представляется слишком «богатым» для получения разумных выводов. В дальнейшем мы будем рассматривать класс

$$
\mathscr{H}(b)=\left\{P \in \mathscr{H}: \sup _{x>K_{*}(P)}\left|c_{F}^{-1} x^{\alpha} F P(X \geqslant x)-1\right| x^{b \alpha_{F}}<\infty\right\}
$$

распределений на $(0 ; \infty)$, где $b>0, K_{*}(P)$ обозначает нижнюю грань носителя распределения, $\alpha_{F} \equiv \alpha_{P}$ есть ПСУХ и $c_{F} \equiv c_{P}$ - константа хвоста распределения.

Если $P \in \mathscr{H}(b)$, то

$$
P(X \geqslant x)=c_{F} x^{-\alpha_{F}}\left(1+O\left(x^{-b \alpha_{F}}\right)\right) \quad(x \rightarrow \infty) .
$$

Задача оценивания ПСУХ эквивалентна задаче оценивания индекса $\alpha$ по выборке независимых одинаково распределенных случайных величин с распределением

$$
F(y) \equiv \mathbf{P}(Y<y)=y^{\alpha} \ell(y) \quad(y>0),
$$

где функция $\ell$ медленно меняется в окрестности нуля. Пусть $\mathscr{F}$ обозначает класс распределений, удовлетворяющих (8). Тогда $\alpha \equiv \alpha_{F}$ является функционалом от $F$ :

$$
\alpha_{F}=\lim _{y \downarrow 0} \frac{\ln F(y)}{\ln y} .
$$


Если $\ell(y)$ стремится к константе (скажем, $\left.c_{F}\right)$ при $y \downarrow 0$, то тогда $c_{F}$ также является функционалом от $F$ :

$$
c_{F}=\lim _{y \downarrow 0} y^{-\alpha_{F}} F(y) .
$$

Отметим, что $\mathscr{L}(Y) \in \mathscr{F}$ тогда и только тогда, когда $\mathscr{L}(1 / Y) \in \mathscr{H}$. Традиция рассмотрения указанной эквивалентной задачи восходит к [7].

Классу $\mathscr{H}(b)$ соответствует следующий непараметрический класс распределений на $(0 ; \infty)$ :

$$
\mathscr{F}(b)=\left\{F \in \mathscr{F}: \sup _{y<K^{*}(F)}\left|c_{F}^{-1} y^{-\alpha_{F}} F(y)-1\right| y^{-b \alpha_{F}}<\infty\right\},
$$

где $b$ - положительное число и $K^{*}(F)$ - правая грань носителя распределения $F$. Функция распределения $F \in \mathscr{F}(b)$ удовлетворяет соотношению

$$
F(y)=c_{F} y^{\alpha_{F}}\left(1+O\left(y^{b \alpha_{F}}\right)\right) \quad(y \rightarrow 0) .
$$

В частности, семейству Парето соответствует класс $\left\{F_{a}\right\}_{a>0}$, где $F_{a}(y)=y^{1 / a}, 0<$ $y \leqslant 1$. Более общим является параметрическое семейство $\left\{F_{\alpha, c}, \alpha>0,0<c \leqslant 1\right\}$, где

$$
F_{\alpha, c}(y)=\left(\frac{y}{c}\right)^{\alpha} \quad(0<y \leqslant c) .
$$

Если $Y_{1}, \ldots, Y_{n}$ - выборка независимых случайных величин, распределенных согласно (11), то оценка максимального правдоподобия

$$
\widehat{a}_{n}^{*} \equiv \frac{1}{\widehat{\alpha}_{n}^{*}}=\ln \left(\max _{i \leqslant n} Y_{i}\right)-n^{-1} \sum_{i=1}^{n} \ln Y_{i}
$$

имеет средне-квадратическую ошибку $\mathbf{E}\left(\widehat{a}_{n}^{*} / a-1\right)^{2}=n^{-1}$. Так как непараметрический класс распределений $\mathscr{F}(b)$ значительно «богаче», точность минимаксного оценивания в $\mathscr{F}(b)$ имеет порядок убывания хуже, чем $n^{-1}$.

Обозначим

$$
r=\frac{b}{1+2 b}
$$

Когда речь идет о функции распределения $F_{i}$, мы полагаем $\alpha_{i}=\alpha_{F_{i}}, a_{i}=1 / \alpha_{i} ; \mathbf{E}_{i}$ означает математическое ожидание по распределению $F_{i}$.

Теорема 1. Для любых $\alpha>0 u c>0$ существуют функиии распределения $F_{0}, F_{1} \in \mathscr{F}(b)$ такие, что $\alpha_{F_{0}}=\alpha, c_{F_{0}}=c^{-\alpha}$ и для любой оченки $\widehat{\alpha}_{n}$ показателя скорости убывания хвоста распределения и оченки $\widehat{a}_{n}$ индекса а имеют место неравенства

$$
\begin{aligned}
& \max _{i \in\{0 ; 1\}} \alpha_{F_{i}}^{r / b} c_{F_{i}}^{r} \mathbf{E}_{i}^{1 / 2}\left(\frac{\widehat{\alpha}_{n}}{\alpha_{F_{i}}}-1\right)^{2} \geqslant\left(\frac{8 r}{n e}\right)^{r} \frac{t_{n}}{2}, \\
& \max _{i \in\{0 ; 1\}} a_{F_{i}}^{-r / b} c_{F_{i}}^{r} \mathbf{E}_{i}^{1 / 2}\left(\frac{\widehat{a}_{n}}{a_{F_{i}}}-1\right)^{2} \geqslant\left(\frac{8 r}{n e}\right)^{r} \frac{t_{n}}{2}
\end{aligned}
$$

npu $n>4 \max \left\{\alpha^{2} c^{-2 \alpha b} ; c^{\alpha} \alpha^{-1 / b}\right\}$, гдe

$$
t_{n}=\left(1-\left(\frac{4}{n}\right)^{r} \alpha^{-r / b} \max _{i \in\{0 ; 1\}}\left(c^{\left.r \alpha_{F_{i}} \vee 1\right)}\right) e^{-1 /(4 n)} .\right.
$$

Согласно (12), для любой оценки $\widehat{\alpha}_{n}$ существует функция распределения $F \in \mathscr{F}(b)$ такая, что

$$
\mathbf{E}_{F}^{1 / 2}\left(\frac{\widehat{\alpha}_{n}}{\alpha_{F}}-1\right)^{2} \geqslant \frac{1}{2}\left(\frac{8 r}{n e}\right)^{r} \alpha_{F}^{-r / b} c_{F}^{-r}(1+o(1)) .
$$


Чем меньше $\alpha_{F}$, тем тяжелее хвост распределения и тем хуже становится нижняя граница $\mathbf{E}_{F}^{1 / 2}\left(\widehat{\alpha}_{n} / \alpha_{F}-1\right)^{2}$. Теорема 1 дает теоретическое обоснование известному мнению, что оценивать характеристики распределений с более тяжелыми хвостами труднее.

Важной особенностью нашего результата является зависимость нижней границы от $\alpha_{F}$ и $c_{F}$. Поэтому предлагаемые границы можно назвать «неравномерными».

Неравенства (12)-(14) показывают, что естественной нормирующей последовательностью для $\widehat{\alpha}_{n} / \alpha_{F}-1$ является

$$
n^{-r} \alpha_{F}^{-r / b} c_{F}^{-r}
$$

(cр. с (6), заметив, что $r \rightarrow 1 / 2$ и $r / b \rightarrow 0$ при $b \rightarrow \infty)$. Более того, «равномерная» нижняя граница была бы бессмысленна: для любой оценки $\widehat{\alpha}_{n}$

$$
\sup _{F \in \mathscr{F}(b)} \mathbf{E}_{F}\left(\frac{\widehat{\alpha}_{n}}{\alpha_{F}}-1\right)^{2} n^{r} \rightarrow \infty \quad(n \rightarrow \infty) .
$$

Число $b$ часто принимает значения 1 (как в случае распределения Фреше) или 2 (как в случае распределения Коши), см. [8], [10]. Следовательно, порядок убывания средне-квадратической ошибки ПСУХ в типичных ситуациях есть $n^{-1 / 3}$ или $n^{-2 / 5}$.

Обозначим через

$$
\widehat{a}_{n}^{\mathrm{RE}}(x)=\frac{\sum_{i=1}^{n} \ln \left(X_{i} / x\right) \mathbf{1}\left\{X_{i} \geqslant x\right\}}{\sum_{i=1}^{n} \mathbf{1}\left\{X_{i} \geqslant x\right\}}, \quad \widehat{\alpha}_{n}^{\mathrm{RE}}(x)=\frac{1}{a_{n}^{\mathrm{RE}}(x)}
$$

RE-оценки ПСУХ $\alpha$ и индекса $a=1 / \alpha$. RE-оценка, по-видимому, является единственной оценкой ПСУХ, для которой асимптотика средне-квадратической ошибки известна:

$$
\mathbf{E}\left(\frac{\widehat{a}_{n}^{\mathrm{RE}}(x)}{a}-1\right)=v(x), \quad \mathbf{E}\left(\frac{\widehat{a}_{n}^{\mathrm{RE}}(x)}{a}-1\right)^{2} \sim \frac{1}{n \mathbf{P}(X \geqslant x)}+v^{2}(x)
$$

в случае независимых одинаково распределенных случайных величин с тяжелым хвостом, где

$$
v(x)=a^{-1} \mathbf{E}\left\{\ln \left(\frac{X}{x}\right) \mid X \geqslant x\right\}-1
$$

[16], [18]. RЕ-оценку ввели Ч. Голди и Р. Смит [11] (см. [8], [11], [18] об оценках константы хвоста распределения). Для RE-оценки $\widehat{a}_{n}^{\mathrm{RE}}\left(x_{n}\right)$ с пороговым параметром $x \equiv x_{n} \sim\left(n /\left(8 r \alpha^{2} c^{\alpha}\right)\right)^{r /(\alpha b)}$ имеем

$$
\max _{i \in\{0 ; 1\}} a_{F_{i}}^{-r / b} c_{F_{i}}^{r} \mathbf{E}_{F_{i}}^{1 / 2}\left(\frac{\widehat{a}_{n}^{\mathrm{RE}}}{a_{F_{i}}}-1\right)^{2} \sim(8 r)^{-r /(2 b)} n^{-r} \quad(n \rightarrow \infty),
$$

где $F_{i}$ есть функция распределения $\mathscr{L}_{i}(1 / Y)$. Правая часть $(18)$ отличается от правой части (13) лишь на множитель $e^{r} / \sqrt{2 r}$.

Пусть $\widehat{c}_{n}$ обозначает произвольную оценку константы хвоста распределения. Следующая теорема предлагает нижнюю границу средне-квадратической ошибки оценки $\widehat{c}_{n}$.

Теорема 2. Для любьх $\alpha>0$ и $>>0$ существуют функиии распределения $F_{0}, F_{1} \in \mathscr{F}(b)$ maкuе, ито $\alpha_{F_{0}}=\alpha, c_{F_{0}}=c^{-\alpha} u$

$$
\max _{i \in\{0 ; 1\}} t_{i, n} \alpha_{F_{i}}^{r / b} c_{F_{i}}^{r} \mathbf{E}_{i}^{1 / 2}\left(\frac{\widehat{c}_{n}}{c_{F_{i}}}-1\right)^{2} \geqslant \frac{r}{2 b}(\ln n)\left(\frac{8 r}{n e}\right)^{r}
$$

для всех достаточно больиих $n$, где $\max _{i \in\{0 ; 1\}}\left|t_{i, n}-1\right| \rightarrow 0$ nри $n \rightarrow \infty$. 
Мы теперь переходим к задаче о нижних границах точности оценивания экстремального квантиля. Мы будем называть квантиль «экстремальным», если уровень $q_{n}$ стремится к нулю с ростом $n$. Разумеется, имеется бесконечное число возможных порядков скорости убывания $q_{n}$. Следующая теорема 3 предлагает нижнюю границу средне-квадратической ошибки оценок экстремального квантиля в случае $q_{n} \asymp n^{-1 /(1+2 b)}$. А именно, рассматриваются квантили уровня $q_{n}=v n^{-1 /(1+2 b)}$, где $v$ отделено от нуля.

Обозначим через

$$
y_{i} \equiv y_{i}\left(q_{n}\right)=F_{i}^{-1}\left(q_{n}\right)
$$

квантиль уровня $q_{n}$. Соответственно, $1 / y_{i}$ есть верхний квантиль распределения $\mathscr{L}_{i}(1 / Y)$. В финансовых приложениях (см., к примеру, [18] и ссылки там) уровень 0.05 может считаться экстремальным, так как эмпирический квантиль уровня $q \leqslant 0.05$ представляется ненадежным.

Теорема 3. Пусть $\widehat{y}_{n}-$ произвольная оченка квантиля уровня $q_{n}$. Для любьх $\alpha>0, c>0 u v<\left(8 \alpha^{2} r c^{-2 \alpha b}\right)^{r / b}$ существуют функиии распределения $F_{0}, F_{1} \in \mathscr{F}(b)$ maкue, ито $\alpha_{F_{0}}=\alpha, c_{F_{0}}=c^{-\alpha} u$

$$
\max _{i \in\{0 ; 1\}} k_{i, n} \alpha_{i}^{r / b} c_{F_{i}}^{r}\left|\ln w_{i}\right|^{-1} \mathbf{E}_{i}^{1 / 2}\left(\frac{\widehat{y}_{n}}{y_{i}}-1\right)^{2} \geqslant \frac{1}{2}\left(\frac{8 r}{n e}\right)^{r}
$$

для всех достаточно больших $n$, где $w_{i}=v^{1 / \alpha_{i}}\left(8 r \alpha_{i}^{2} c_{F_{i}}^{2 b}\right)^{-r /\left(\alpha_{i} b\right)} u \max _{i \in\{0 ; 1\}} \mid k_{i, n}-$ $1 \mid \rightarrow 0$ при $n \rightarrow \infty$. Неравенство (20) останется в силе, если $\widehat{y}_{n} / y_{i}-1$ в его левой части заменить на $y_{i} / \widehat{y}_{n}-1$.

Чем меньше $v$, тем меньше $q_{n}$ и, следовательно, тем труднее задача оценивания. Неравенство (20) согласуется с этим: $\left|\ln w_{i}\right|$ растет с убыванием $v$, увеличивая нижнюю границу.

Заметим, что $1 / \widehat{y}_{n}$ является оценкой верхнего квантиля уровня $q_{n}$ PТХ $\mathscr{L}(1 / Y)$.

3. Доказательства. Пусть $\mathscr{P}$ - некоторое семейство распределений. Приводимая ниже лемма 1 посвящена общей задаче оценивания функционала (характеристики) $a_{P}$ неизвестного распределения $P \in \mathscr{P}$ по выборке $X_{1}, \ldots, X_{n}$ независимых одинаково распределенных случайных величин.

Мы будем считать, что функционал $a_{P}$ является элементом метрического пространства $(\mathscr{X}, d)$. Оценкой $\widehat{a}$ числа $a_{P}$ называется измеримая функция $X_{1}, \ldots, X_{n}$, принимающая значения в подпространстве $\left\{a_{P}: P \in \mathscr{P}\right\} \subset \mathscr{X}$.

Если даны два распределения $\left\{P_{0}, P_{1}\right\} \in \mathscr{P}$, мы обозначаем

$$
a_{i}=a_{P_{i}}, \quad 2 \delta=d\left(a_{0} ; a_{1}\right),
$$

$\mathbf{E}_{i} \equiv \mathbf{E}_{P_{i}}$ есть математическое ожидание по распределению $P_{i}$. Пусть $R$ обозначает функцию потерь, а $d_{\mathrm{H}}\left(P_{0} ; P_{1}\right)$ есть расстояние Хеллингера.

Лемма 1. Если функиия $R^{1 / 2}$ выпукла, то для любой оченки $\widehat{a}$

$$
\max _{i \in\{0 ; 1\}} \mathbf{E}_{i} R\left(d\left(\widehat{a} ; a_{i}\right)\right) \geqslant R(\delta)\left(1-d_{\mathrm{H}}^{2}\right)^{2 n} .
$$

В частности, для квадратичной функции потерь имеем

$$
\max _{i \in\{0 ; 1\}} \mathbf{E}_{i}^{1 / 2} d^{2}\left(\widehat{a} ; a_{i}\right) \geqslant \delta\left(1-d_{\mathrm{H}}^{2}\right)^{n} .
$$

См. [9], [13], [22] и ссылки там касательно литературы по минимаксным нижним границам.

Д ок аз а те льс тв о лем мы 1. Можно предполагать, что $a_{1} \neq a_{0}$. Обозначим

$$
\mathbf{E}_{*}=\max _{i \in\{0 ; 1\}} \mathbf{E}_{i} R\left(d\left(\widehat{a} ; a_{i}\right)\right)
$$


и напомним, что

$$
d_{\mathrm{H}}^{2}\left(P_{0} ; P_{1}\right)=\frac{1}{2} \int\left(f_{0}^{1 / 2}-f_{1}^{1 / 2}\right)^{2}=1-\int \sqrt{f_{0} f_{1}},
$$

где $f_{i}$ - плотность распределения $P_{i}$ по отношению к некоторой мере (например, $P_{0}+$ $\left.P_{1}\right)$. Пусть $f_{i, n}$ обозначает плотность распределения $\mathscr{L}_{i}\left(X_{1}, \ldots, X_{n}\right)$. По неравенству треугольника,

$$
\delta \leqslant \frac{d\left(a_{0} ; \widehat{a}\right)}{2}+\frac{d\left(\widehat{a} ; a_{1}\right)}{2} .
$$

Так как функция $R^{1 / 2}$ выпукла, то $R^{1 / 2}(\delta) \leqslant R^{1 / 2}\left(d\left(\widehat{a} ; a_{0}\right)\right) / 2+R^{1 / 2}\left(d\left(a_{1} ; \widehat{a}\right)\right) / 2$. Поэтому

$$
2 R^{1 / 2}(\delta)\left(1-d_{\mathrm{H}}^{2}\right)^{n} \leq \int R^{1 / 2}\left(d\left(\widehat{a} ; a_{0}\right)\right) \sqrt{f_{0, n}} \sqrt{f_{1, n}}+\int R^{1 / 2}\left(d\left(a_{1} ; \widehat{a}\right)\right) \sqrt{f_{0, n}} \sqrt{f_{1, n}} .
$$

Это соотношение и неравенство Коши-Буняковского влекут

$$
2 R^{1 / 2}(\delta)\left(1-d_{\mathrm{H}}^{2}\right)^{n} \leq \mathbf{E}_{0}^{1 / 2} R\left(d\left(\widehat{a} ; a_{0}\right)\right)+\mathbf{E}_{1}^{1 / 2} R\left(d\left(\widehat{a} ; a_{1}\right)\right) \leq 2 \mathbf{E}_{*}^{1 / 2},
$$

откуда следует (21). Лемма 1 доказана.

Наш подход к задаче получения нижних границ использует два распределения $P_{0}$ и $P_{1}$, где $P_{0}$ - распределение Парето, а $P_{1} \equiv P_{1, n}$ есть «видоизмененная» версия $P_{0}$. Затем мы применяем лемму 1 , которая предоставляет неасимптотическую нижнюю границу точности оценивания при выборе из двух близких альтернатив.

Д о к а з а т е л ь с т в о т е о р е м ы 1 . Пусть заданы произвольные $\alpha>0$ и $c>0$. Мы будем иметь дело с функциями распределения $F_{0}$ и $F_{1}$, где

$$
\begin{aligned}
& F_{0}(y)=\left(\frac{y}{c}\right)^{\alpha} \mathbf{1}\{0<y \leqslant c\}, \\
& F_{1}(y)=\left(\frac{h}{c}\right)^{-\gamma}\left(\frac{y}{c}\right)^{\alpha_{1}} \mathbf{1}\{0<y \leq h\}+\left(\frac{y}{c}\right)^{\alpha} \mathbf{1}\{h<y \leqslant c\},
\end{aligned}
$$

$\alpha_{1}>\alpha, h \in(0 ; c)$. Легко заметить, что $F_{1} \leqslant F_{0}$ и

$$
\alpha_{F_{0}}=\alpha, \quad \alpha_{F_{1}}=\alpha_{1}, \quad c_{F_{0}}=c^{-\alpha}, \quad c_{F_{1}}=c^{-\alpha} h^{-\gamma} .
$$

Обозначим $\alpha_{0}=\alpha$, и пусть

$$
\alpha_{1}=\alpha+\gamma, \quad \gamma=h^{\alpha b} .
$$

Очевидно, что $F_{0} \in \mathscr{F}(b)$. Проверим теперь, что $F_{1} \in \mathscr{F}(b)$. Так как

$$
c_{F_{1}}^{-1} y^{-\alpha_{1}} F_{1}(y)=y^{-\gamma} h^{\gamma} \quad(h<y \leqslant c),
$$

имеем

$$
\sup _{0<y \leqslant c}\left|1-c_{F_{1}}^{-1} y^{-\alpha_{1}} F_{1}(y)\right| y^{-b \alpha_{1}}=\sup _{h<y \leq c}\left(1-y^{-\gamma} h^{\gamma}\right) y^{-b \alpha_{1}} .
$$

Функция в правой части (24) достигает своего максимума на $y_{0}=h\left(1+\gamma /\left(b \alpha_{1}\right)\right)^{1 / \gamma}$ и не превосходит $e^{1 /(e \alpha)} /(b \alpha)$.

Легко проверить, что

$$
d_{\mathrm{H}}^{2}\left(F_{0} ; F_{1}\right)=\left(\frac{h}{c}\right)^{\alpha}\left(1-\frac{\sqrt{1+\gamma / \alpha}}{1+\gamma /(2 \alpha)}\right) \sim \frac{\gamma^{1 / r}}{8 \alpha^{2} c^{\alpha}}
$$

при $\gamma \rightarrow 0$, и

$$
d_{\mathrm{H}}^{2}\left(F_{0} ; F_{1}\right) \leqslant \frac{\gamma^{1 / r}}{8 \alpha^{2} c^{\alpha}}
$$


Неравенство (25) типично в задачах непараметрического оценивания. Непараметрический класс распределений обычно настолько «богат», что можно найти распределения $\left\{P_{t}, t \geqslant 0\right\}$ такие, что $d_{\mathrm{H}}\left(P_{0} ; P_{t}\right) \asymp|t|^{\nu}$, где $\nu>1$, в то время как в случае регулярного параметрического семейства $d_{\mathrm{H}}\left(P_{0} ; P_{t}\right) \asymp|t|$.

Согласно лемме 1 ,

$$
\max _{i \in\{0 ; 1\}} \mathbf{E}_{i}^{1 / 2}\left(\widehat{\alpha}_{n}-\alpha_{F_{i}}\right)^{2} \geqslant \frac{\gamma}{2}\left(1-d_{\mathrm{H}}^{2}\right)^{n} \geqslant \frac{\gamma}{2}\left(1-\frac{\gamma^{1 / r}}{8 \alpha^{2} c^{\alpha}}\right)^{n} .
$$

Максимум по $\gamma$ в этом неравенстве достигается на $\gamma=\gamma_{n}$, где

$$
\gamma_{n} \equiv \gamma_{n}(\alpha, b, c)=\frac{\left(8 \alpha^{2} c^{\alpha}\right)^{r}}{(1+n / r)^{r}}
$$

и мы получим

$$
\max _{i \in\{0 ; 1\}} \mathbf{E}_{i}^{1 / 2}\left(\widehat{\alpha}_{n}-\alpha_{i}\right)^{2} \geqslant \frac{1}{2}\left(\frac{8 r \alpha^{2} c^{\alpha}}{n}\right)^{r}\left(1+\frac{r}{n}\right)^{-n-r} .
$$

Заметим, что $h<c$ при $n \geqslant 8 \alpha^{2} r c^{-2 b \alpha}$. Легко проверить, что $\alpha / \alpha_{1}=1-\gamma / \alpha_{1}$, $\gamma=\gamma_{n} \leqslant\left(4 \alpha^{2} c^{\alpha} / n\right)^{r}$ и $c^{\alpha r} \leqslant c^{\alpha_{1} r}$ при $c>1$. Следовательно,

$$
\left(\frac{\alpha}{\alpha_{1}}\right)^{2 r} \geqslant 1-\frac{2 r \gamma}{\alpha_{1}} \geqslant 1-2 r\left(\frac{8 r}{n}\right)^{r} \alpha_{1}^{2 r-1} c^{\alpha r} \geqslant 1-\left(\frac{4}{n}\right)^{r} \alpha_{1}^{2 r-1}\left(c^{r \alpha_{1}} \vee 1\right) .
$$

Принимая во внимание (23), мы приходим к (12). Аналогично $\left(a_{i}:=1 / \alpha_{i}, a:=a_{0}\right)$,

$$
\max _{i \in\{0 ; 1\}} \mathbf{E}_{i}^{1 / 2}\left(\widehat{a}_{n}-a_{i}\right)^{2} \geqslant \frac{a a_{1}}{2} \gamma\left(1-\frac{\gamma^{1 / r} a^{2}}{8 c^{1 / a}}\right)^{n}=\frac{a_{1}}{2} a^{1-2 r} c^{r / a}\left(\frac{8 r}{n}\right)^{r}\left(1+\frac{r}{n}\right)^{-n-r},
$$

что влечет (13). Теорема доказана.

Что касается (18), если $F_{i}$ есть функция распределения случайной величины $1 / X$, $i \in\{0 ; 1\}$, то представляется естественным положить

$$
x_{n}=\frac{1}{h}=\gamma_{n}^{-a / b} \sim\left(\frac{n}{8 r \alpha^{2} c^{\alpha}}\right)^{r /(\alpha b)} .
$$

Тогда $v\left(x_{n}\right)=0$ ввиду $(17)$, и (18) следует из (16).

Д ок аз а т ель с т в о т е о р е мы 2. Обозначим $c_{i}:=c_{F_{i}}$, где функции распределения $F_{0}$ и $F_{1}$ - те же, что и раньше. Тогда

$$
c_{F_{1}}-c_{F_{0}}=c^{-\alpha}\left(\gamma^{-\gamma /(\alpha b)}-1\right) \geqslant \frac{c^{-\alpha} \gamma|\ln \gamma|}{\alpha b} .
$$

Используя это неравенство и (22), мы выводим

$$
\max _{i \in\{0 ; 1\}} \mathbf{E}_{i}^{1 / 2}\left(\widehat{c}_{n}-c_{i}\right)^{2} \geqslant c^{-\alpha}(2 \alpha b)^{-1} \gamma|\ln \gamma|\left(1-\frac{\gamma^{1 / r}}{8 \alpha^{2} c^{\alpha}}\right)^{n} .
$$

Полагая $\gamma=\gamma_{n}$ (см. (27)), получим

$$
\max _{i \in\{0 ; 1\}} \mathbf{E}_{i}^{1 / 2}\left(\widehat{c}_{n}-c_{i}\right)^{2} \geqslant r c^{\alpha(r-1)}\left(2 \alpha^{r / b} b\right)^{-1}\left(\frac{8 r}{n e}\right)^{r} \ln \left(\frac{n}{8 r \alpha^{2} c^{\alpha}}\right)
$$

для всех достаточно больших $n$, откуда и следует (19). Теорема 2 доказана.

Д ок аз а т ель с т в о т е о ре мы 3. Обозначим

$$
\kappa=v^{1 / \alpha}\left(8 \alpha^{2} c^{\alpha} r\right)^{-r /(\alpha b)} \text {. }
$$


Нам будет удобно иметь дело с эквивалентной задачей оценивания квантилей уровня

$$
q_{n}=\kappa^{\alpha} \gamma_{n}^{1 / b} \sim v n^{-r / b},
$$

где $\gamma=\gamma_{n}$ определено в (27).

Заметим, что $q_{n}=(\kappa h)^{\alpha}$. Пусть функции распределения $F_{0}, F_{1}$ - те же, что и раньше. Легко проверить, что

$$
y_{0}=c \kappa h=c q_{n}^{1 / \alpha}<h, \quad y_{1}=(c \kappa)^{\alpha / \alpha_{1}} h, \quad c \kappa<1 .
$$

Поскольку $e^{x}-1 \geqslant x e^{x / 2}(x \geqslant 0)$, мы выводим

$$
\begin{aligned}
y_{1}-y_{0} & =c q_{n}^{1 / \alpha_{1}}\left(\left(\frac{h}{c}\right)^{\gamma / \alpha_{1}}-q_{n}^{\gamma /\left(\alpha \alpha_{1}\right)}\right) \\
& =\left((c \kappa)^{-\gamma / \alpha_{1}}-1\right) c \kappa h \geqslant \gamma^{1+1 /(\alpha b)}(c \kappa)^{1-\gamma /\left(2 \alpha_{1}\right)} \frac{|\ln c \kappa|}{\alpha_{1}} .
\end{aligned}
$$

Это соотношение и (22) влекут

$$
\max _{i \in\{0 ; 1\}} \mathbf{E}_{i}^{1 / 2}\left(\widehat{y}_{n}-y_{i}\right)^{2} \geqslant \frac{|\ln c \kappa|}{2 \alpha_{1}} c \kappa \gamma^{1+1 /(\alpha b)}\left(1-\frac{\gamma^{1 / r}}{8 \alpha^{2} c^{\alpha}}\right)^{n} .
$$

Полагая $\gamma=\gamma_{n}$, получим

$$
\begin{aligned}
\max _{i \in\{0 ; 1\}} \mathbf{E}_{i}^{1 / 2}\left(\frac{\widehat{y}_{n}}{y_{i}}-1\right)^{2} & \geqslant \frac{1}{y_{i}} \frac{|\ln c \kappa|}{2 \alpha_{1}} c \kappa \gamma^{1+1 /(\alpha b)}\left(1-\frac{\gamma^{1 / r}}{8 \alpha^{2} c^{\alpha}}\right)^{n} \\
& =\frac{|\ln c \kappa|}{2 \alpha_{1}}(c \kappa)^{1-\alpha / \alpha_{i}} \gamma_{n}\left(1+\frac{r}{n}\right)^{-n} .
\end{aligned}
$$

Используя (27) и (28), приходим к соотношению

$$
\max _{i \in\{0 ; 1\}} k_{i, n} \alpha_{i}^{r / b} c_{F_{i}}^{r} \mathbf{E}_{i}^{1 / 2}\left(\frac{\widehat{y}_{n}}{y_{i}}-1\right)^{2} \geqslant \frac{1}{2}|\ln c \kappa|\left(\frac{8 r}{n e}\right)^{r}
$$

для всех достаточно больших $n$. Заметим, что

$$
c \kappa=v^{1 / \alpha}\left(8 \alpha^{2} r c_{F_{0}}^{2 b}\right)^{-r /(\alpha b)} .
$$

Следовательно, (20) вытекает из (30).

Поскольку

$$
\left|1 / y_{1}-1 / y_{0}\right|=\left|y_{1}-y_{0}\right| /\left(y_{0} y_{1}\right) \geqslant|\ln c \kappa|(c \kappa)^{-1+\gamma /\left(2 \alpha_{1}\right)} \gamma^{1-1 / \alpha b} / \alpha_{1},
$$

мы имеем

$$
\max _{i \in\{0 ; 1\}} \mathbf{E}_{i}^{1 / 2}\left(\frac{y_{i}}{\widehat{y}_{n}}-1\right)^{2} \geqslant \frac{|\ln c \kappa|}{2 \alpha_{1}}(c \kappa)^{\gamma /\left(2 \alpha_{1}\right)} \gamma\left(1-\frac{\gamma^{1 / r}}{8 \alpha^{2} c^{\alpha}}\right)^{n},
$$

откуда вытекает следующий аналог неравенства (20): в условиях теоремы 3

$$
\max _{i \in\{0 ; 1\}} k_{i, n} \alpha_{i}^{r / b} c_{F_{i}}^{r} \mathbf{E}_{i}^{1 / 2}\left(\frac{y_{i}}{\widehat{y}_{n}}-1\right)^{2} \geqslant \frac{1}{2}|\ln c \kappa|\left(\frac{8 r}{n e}\right)^{r}
$$

и, следовательно,

$$
\max _{i \in\{0 ; 1\}} k_{i, n} \alpha_{i}^{r / b} c_{F_{i}}^{r}\left|\ln w_{i}\right|^{-1} \mathbf{E}_{i}^{1 / 2}\left(\frac{y_{i}}{\widehat{y}_{n}}-1\right)^{2} \geqslant \frac{1}{2}\left(\frac{8 r}{n e}\right)^{r}
$$

для всех достаточно больших $n$. Неравенство (31) является минимаксной нижней границей средне-квадратической ошибки произвольной оценки $1 / \widehat{y}_{n}$ верхнего квантиля уровня $q_{n}$ распределений $\mathscr{L}_{i}(1 / Y) \in \mathscr{H}(b)$. Теорема 3 доказана.

Автор признателен рецензентам за полезные замечания. 


\section{СПИСОК ЛИТЕРАТУРЫ}

1. Beirlant J., Bouquiaux C., Werker B. J. M. Semiparametric lower bounds for tail index estimation. - J. Statist. Plann. Inference, 2006, v. 136, № 3, p. 705-729.

2. Devroye L. Another proof of a slow convergence result of Birgé. - Statist. Probab. Lett., 1995, v. 23, № 1, p. 63-67.

3. Donoho D. L., Liu R. C. Geometrizing rates of convergence. II, III. - Ann. Statist., 1991, v. 19, № 2 , p. $633-667$; p. 668-701.

4. Drees $H$. Minimax risk bounds in extreme values theory. - Ann. Statist., 2001, v. 29, № 1 , p. $266-294$.

5. Fama E.F., Roll R. Some properties of symmetric stable distributions. - J. Amer. Statist. Assoc., 1968, v. 63, p. 817-836.

6. Farrell R. H. On the best obtainable asymptotic rates of convergence in estimation of a density function at a point. - Ann. Math. Statist., 1972, v. 43, p. 170-180.

7. Hall $P$. On estimating the endpoint of a distribution. - Ann. Statist., 1982, v. 10, № 2 , p. 556-568.

8. Hall P., Welsh A. H. Best attainable rates of convergence for estimates of parameters of regular variation. - Ann. Statist., 1984, v. 12, № 3, p. 1079-1084.

9. Huber C. Lower bounds for function estimation. - Festschrift for L. Le Cam. New York: Springer, 1997, p. 245-258.

10. Embrechts P., Klüppelberg C., Mikosch T. Modelling Extremal Events for Insurance and Finance. Berlin: Springer-Verlag, 1997, 645 p.

11. Goldie C.M., Smith R.L. Slow variation with remainder: theory and applications. Quart. J. Math. Oxford, 1987, v. 38, № 149, p. 45-71.

12. Ибрагимов И. А., Хасьминский Р. З. Об оценке плотности распределения. - Зап. науч. сем. ЛОМИ, 1980, т. 98, с. 61-85.

13. Ибрагимов И. А., Хасьминский Р. З. Асимптотическая теория оценивания. М.: Наука, 1979, 527 с.

14. Lenstra A. J. Cramér-Rao revisited. - Bernoulli, 2005, v. 11, № 2, p. 263-282.

15. Mandelbrot B. B. New methods in statistical economics. - J. Political Economy, 1963, v. 71, p. $421-440$.

16. Новак С.Ю., Утев С.А. Об асимптотике распределения отношения сумм случайных величин. - Сиб. матем. журн., 1990, т. 31, с. 92-101.

17. Новак С. Ю. О распределении отношения сумм случайных величин. - Теория вероятн. и ее примен., 1996, т. 41, в. 3, с. 533-560.

18. Novak $S . Y$. Advances in extreme value theory with applications to finance. - New Business and Finance Research Developments. Ed. by C. Keene. New York: Nova Science, 2009, p. 199-251.

19. Pfanzagl J. On local uniformity for estimators and confidence limits. - J. Statist. Plann. Inference, 2000, v. 84, № 1-2, p. 27-53.

20. Resnick S.I. Heavy tail modeling and teletraffic data. - Ann. Statist., 1997, v. 25, № 5, p. 1805-1869.

21. Шемякин А.Е. Об информационных неравенствах в теории параметрического оценивания. - Теория вероятн. и ее примен., 1992, т. 37, в. 3, с. 121-123.

22. Tsybakov A. B. Introduction to Nonparametric Estimation. New York: Springer, 2009, $175 \mathrm{p}$.

Поступила в редакцию 20.XII.2009

Исправленный вариант 3.III.2013 